\title{
Continuous dispersal in a model of predator-prey-subsidy population dynamics
}

\author{
Alastair Bassett, Andrew L. Krause, and Robert A. Van Gorder* \\ Mathematical Institute, University of Oxford, Andrew Wiles Building, Radcliffe Observatory Quarter, Woodstock Road, Oxford, OX2 6GG, UK \\ ${ }^{*}$ Robert.VanGorder@maths.ox.ac.uk
}

\begin{abstract}
Spatial dispersion and clustering of populations is important for a complete understanding of ecological dynamics in many ecosystems. Motivated by an Arctic ecosystem involving foxes (predator), lemmings (prey), and seal carrion (subsidy), we extend the spatially homogeneous (island) model of predator-prey-subsidy dynamics originally proposed in [A.L. Nevai and R.A. Van Gorder. Journal of Biological Dynamics, 6:891-922, 2012] to account for dispersion of predator and prey populations throughout a twodimensional domain. Our model has qualitatively similar behaviours in large regions of parameter space to the aforementioned island model. Quantitative effects due to varying the rate of dispersal and restricting the spatial extent of the subsidy are demonstrated. We observe nontrivial clustering of the predator and prey populations due to the presence of the subsidy only in a specific subregion of the domain, which suggests a novel mechanism of spatial partitioning and pattern formation due to the presence of subsidy in only part of the region of interest.
\end{abstract}

Keywords: Spatial population dynamics, Allochthonous resource subsidy, Predator-prey dynamics

\section{Introduction}

Mathematical models have played a significant role in understanding the dynamics of populations for over a century. These models have helped abstract key features of interactions between biological organisms that have given insight into a variety of observed phenomena in real populations. Recently models have been employed to explain the role of subsidy food resources on predator-prey dynamics $[11,16]$, as well as more complicated subsidy-mediated interactions between species [5]. These resources are considered allochthonous as they are the waste or remains of other organisms that may be part of other population dynamics, and so the interactions are different from more traditional predator-prey, cooperative, or competitive dynamics.

Predator-prey systems have been known to exhibit a number of different types of behaviour, including species extinctions, coexistence, temporal oscillations in populations [12, 27, 8] and chaotic dynamics $[11,25,13]$. For some predators, there exists a resource subsidy which they can use as an alternative food source to their favoured prey. It was suggested in [14] that the presence of a subsidy could stabilise population equilibria, which is supported by more recent studies [26, 2, 15]. One canonical example of these dynamics is the interaction between arctic fox (Alopex lagopus) and lemming (Cricetidae family) populations. In coastal arctic regions, arctic foxes are known to subsist both on lemmings and on seal (Phocidae family) carcasses, which act as a resource subsidy. The seal carcasses are deposited onto sea ice after predation by polar bears $(U r$ sus maritimus)[20]. The foxes and lemmings have been known to have large ranges of movement $[4,17]$, whereas the seal carcasses, which drift with the sea ice, have limited ranges of movement.

Many models used to analyse predator-prey and predatorprey-subsidy interactions assume that the populations exist in the same location [14, 12, 13]. Holt [6] suggested a model for two prey species separated into two patches with a common predator which can migrate between both patches. Separation of the two prey populations was found to have a stabilising effect on both the prey and predator populations. However, under certain conditions, one of the prey populations could be vulnerable to extinction due to predator population increases in the other patch. This is supported by the predictions of the two patch predator-prey-subsidy model in [16], in which a heavily subsidised predator could force prey extinction. This model was extended in [11] to include effects of seasonality, as well as an $n$-patch (or, "stepping-stone") model allowing for coarsegrained spatial detail. This multiple patch model is useful in understanding some long and short-range effects, such as increased mortality rates during migration.

We shall extend these ideas of spatial movement previously considered in multipatch models by allowing continuous dispersal of the predator and prey populations over a spatial region. This was originally suggested in [16]. These spatial effects are crucial to understanding population dynamics in many settings [1]. Reviews and comparison of continuous and discrete spatial models for population growth and predator-prey dynamics can be found in $[23,7]$. We contribute to these discussions by considering spatially heterogeneous subsidy distributions, focusing on the potential they have for destabilizing coexistence equilibria between predator-prey interactions, leading to ecological cascade effects. Understanding the dynamics of varying subsidy spatial distributions has implications for anthropogenic influences on predator-prey interactions, such as their ability to 
decouple predator-prey dynamics [19], in addition to implications for conservation efforts such as the introduction of additional subsidy in the Arctic ecosystem mentioned before.

In Section 2, we examine a partial differential equation model for the interactions between a predator population (e.g. arctic foxes), a prey population (e.g. lemmings) and a resource subsidy (e.g. seal carcasses), where all three are able to move throughout a continuous spatial domain. We analyze this model numerically in Section 3, highlighting the effects of various parameters in the model. The biological implications of our results, and a comparison with island and patch models, are then discussed in Section 4. Conclusions follow in Section 5.

\section{Continuous Dispersal in Predator-Prey-Subsidy Interac- tions}

In [16], a two patch system was considered, where one patch contains only the prey, and the other contains only the subsidy. The predators were free to move between the patches, at a rate proportional to the difference between the predator populations in each patch. This model was seen to sustain coexistence, limit cycles, or extinction of either the prey or the predator, depending on the parameters.

As suggested in [16], a more realistic model could account for continuous spatial variation in the populations. This motivates us to treat the prey, subsidy and predator populations $x$, $s$ and $y$ as functions of both space and time. We assume that these populations disperse randomly over a spatial region $\Omega$. This leads to the following set of reaction-diffusion equations:

$$
\begin{aligned}
& \frac{\partial x}{\partial t}=d_{1} \Delta x+r x\left(1-\frac{x}{k}\right)-\theta\left(\frac{x}{x+a}\right) y, \\
& \frac{\partial s}{\partial t}=d_{2} \Delta s+I-\gamma s-\psi\left(\frac{s}{s+c}\right) y, \\
& \frac{\partial y}{\partial t}=d_{3} \Delta y+\epsilon \theta\left(\frac{x}{x+a}\right) y+\eta \psi\left(\frac{s}{s+c}\right) y-\delta y,
\end{aligned}
$$

where $x=x(\alpha, \beta, t)$ and $y=y(\alpha, \beta, t)$ are the prey and predator populations, $s=s(\alpha, \beta, t)$ is the quantity of subsidy, $t$ is time, $(\alpha, \beta) \in \Omega$ are the spatial variables, $r, k, \theta, a, I, \gamma, \psi, c, \epsilon, \eta>0$ are all non-negative kinetic parameters, and $d_{1}, d_{2}$ and $d_{3}$ are positive diffusion coefficients for the three populations.

In all of our simulations we take spatially uniform initial conditions to match those in [11]. So we have $x(\alpha, \beta, 0)=0.2$, $s(\alpha, \beta, 0)=0$, and $y(\alpha, \beta, 0)=0.1$. We also assume that populations cannot leave the domain so if $\boldsymbol{n}$ is the outward normal vector to the domain we have,

$$
\boldsymbol{n} \cdot \nabla x=0, \quad \boldsymbol{n} \cdot \nabla s=0, \quad \boldsymbol{n} \cdot \nabla y=0 \quad \text { for all }(\alpha, \beta) \in \partial \Omega,
$$

where $\partial \Omega$ denotes the boundaries of the domain $\Omega$.

In Equation 1, the second term on the right hand side accounts for logistic growth, where $r$ is the intrinsic growth rate and $k$ is the carrying capacity of the prey. The final term accounts for predation, with $\theta$ as the maximum predation rate of the prey, and $a$ as the half-saturation constant. Equation 2 describes changes in subsidy populations. The rate at which the subsidy appears is $I$, whereas $\gamma$ is the rate at which it disappears

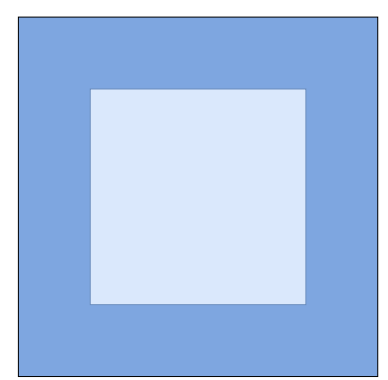

Figure 1: The spatial domain $\Omega$. The darker region around the outside is $\Omega_{0}$, on which the subsidy increases. We will use $A$ to denote the ratio of the area of the darker region to the area of the entire outer square.

or becomes inedible. As with the prey, the final term accounts for predation, where $\psi$ is the maximum rate of predation and $c$ is the half-saturation constant. Equation 3 models the behaviour of the predator populations. The second and third terms on the right hand side account for predation of the prey and the subsidy respectively, with $\epsilon$ and $\eta$ being the conversion factors of the prey and the subsidy. The mortality rate of the predator is given by $\delta$.

For a realistic modelling of populations in the arctic, the area on which the amount of subsidy increases should be restricted. As is discussed in [20], seal carcasses are deposited around coastal regions of the arctic. In the system of PDEs considered here, we will reflect this by defining the subsidy creation rate $I$ as

$$
I(\alpha, \beta)= \begin{cases}i & \text { if }(\alpha, \beta) \in \Omega_{0}, \\ 0 & \text { if }(\alpha, \beta) \notin \Omega_{0},\end{cases}
$$

where $i$ is the rate at which the subsidy is created per unit area, and $\Omega_{0}$ is some subset of $\Omega$. To avoid numerical artifacts, and to incorporate drift due to sea ice, we do assume that $d_{2}>0$. However, as we think of the subsidy as consisting entirely of detritus (e.g. seal carrion) we assume it disperses very little and so take $d_{2} \ll d_{1}, d_{3}$.

In the data presented in Section 3, we will take the region $\Omega$ to be a square of nondimensional unit length, and the region $\Omega_{0} \subseteq \Omega$ on which the subsidy increases to be along the boundaries of $\Omega$, as shown in Figure 1. The region where the subsidy is not being introduced, $(\alpha, \beta) \notin \Omega_{0}$, is the inner square in Figure 1 and can be thought of as the Arctic mainland in the foxlemming-seal carrion ecosystem. We introduce the parameter $A=\left|\Omega_{0}\right| /|\Omega|$ to measure the area of the region where subsidy is being introduced, and vary it systematically in our simulations.

To facilitate comparison with island and stepping-stone models, we use the following notation for the spatial average and equilibrium values of each population. The population density of the prey throughout the domain, $x(\alpha, \beta, t)$, can be averaged via

$$
\bar{x}(t)=\frac{1}{|\Omega|} \int_{\Omega} x(\alpha, \beta, t) d \alpha d \beta .
$$

Similarly we let $x^{*}(\alpha, \beta)$ denote the equilibrium population density and $\bar{x}^{*}$ the spatially averaged equilibrium. Similar notation is used for the average of the predator $y$ and the subsidy $s$.

This model poses a number of unique challenges not present in ODE models of predator-prey subsidy dynamics. PDEs 


\section{$\begin{array}{lllll}0 & 0 & 0 & 0 & 0 \\ 0 & 0 & 0 & 0 & 0 \\ 0 & 0 & 0 & 0 & 0 \\ 0 & 0 & 0 & 0 & 0 \\ 0 & 0 & 0 & 0 & 0\end{array}$}

(a)

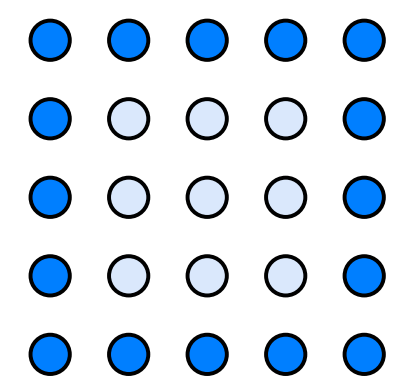

(b)

Figure 2: Discretizing $\Omega$ as a $5 \times 5$ grid gives four symmetric possibilities for $\Omega_{0}$ : the whole of $\Omega$, the possibilites shown in (a) and (b), where the darker dots make up $\Omega_{0}$, and the empty set. For much larger grids we anticipate that $\Omega_{0}$ can be varied as a closer approximation to a continuous subset.

are more difficult to analyze numerically as there are multiple sources of error to account for due to spatial and temporal discretizations. Steady state analysis is also more challenging as at steady state, we are still left with a system of coupled PDEs in two spatial dimensions. The equations depend on the spatial variables $\alpha$ and $\beta$ implicitly through the populations $x, s$ and $y$, but also explicitly through the heterogeneous specification of $I$, the subsidy creation rate. Another important aspect of the model is the nonlinearity of the system. Diffusion has been known to drive instability in nonlinear systems [24], and solutions to similar models, such as the $n$-patch model in [11] have been known to exhibit chaotic behaviour due to temporal forcing. Hence there are a variety of different behaviours that Equations (1)-(3) could exhibit. We are particularly interested in behaviours not present in the previous spatially discrete models.

In our numerical simulations we take $r=0.1, \theta=5, a=1$, $\gamma=1, \psi=5, c=1$ and $\delta=0.1$. We also set the subsidy diffusion $d_{2}=0.0001$ and the prey and predator diffusion rates to be equal and denote them by $d=d_{1}=d_{3}$. We systematically vary $d, k, I, \epsilon, \eta$ to explore model behaviours.

\section{Numerical Simulations}

We discretize the spatial derivatives of Equations (1)-(3) using the 5-point Laplacian on a fixed square grid. If we do not discretize time, and hence consider a system of coupled ODEs, this is equivalent to a formulation of the model in terms of patches. For a coarse discretization, such as in Figure 2, we would expect model behaviour to be comparable to the $n$ patch model described in [11]. As we are interested in spatially continuous dynamics, we considered the size of all grids in our simulations to be at least $50 \times 50$ grid points or larger, typically taking $100 \times 100$, and ensured convergence in the spatial step size for fixed points in time $t$. This allows us to reasonably assume that the model behaviours we observe are ones corresponding to the spatially continuous dynamics in the limit of the number of grid points tending to infinity.

To perform the simulations, we then made use of the MATLAB function 'ode45' [21]. We constrained the maximum time-step for this process to be at most $10^{-2}$. For each change in qualitative behaviour, we checked that our results converged in the time step. We also reduced the maximum time step and error tolerances when local populations were observed to be small and of the same order as the time step. This allows us to be certain that limit cycle behaviour is observed, as opposed to one population being driven into extinction.

In order to calculate the spatially averaged populations $\bar{x}$ and $\bar{y}$, we numerically approximated the integrals defined by (5) using simple quadratures. As our spatial grids were fine, the errors from discretizing these integrals are smaller than the local truncation errors introduced from the spatial discretization of the PDEs.

We present solutions to our model for three combinations $(k, i)$ of the prey carrying capacity and the rate of subsidy increase. These are $(0.1,0.1),(0.4,0.3)$ and $(2.4,0.1)$. In the spatially homogeneous case, corresponding to $\Omega=\Omega_{0}$, or $A=1$, these correspond to different sets of dynamics depending on the values of $\eta$ and $\epsilon$. However, we shall in general be interested in the heterogeneous case of $0<A<1$, and shall be able to obtain numerical results. In the first case, we see predator extinction, prey extinction and coexistence as we vary $\epsilon$ and $\eta$, the conversion rates of the prey and subsidy. For $(k, i)=(0.4,0.3)$, we see coexistence and prey extinction as the conversion rates vary. In this case, we also consider the effects of changing the area upon which subsidy amounts increase. When $(k, i)=(2.4,0.1)$, we see the emergence of limit cycles in the populations. In all of our simulations we normalize the results with respect to the initial conditions $x(\alpha, \beta, 0)=0.2, s(\alpha, \beta, 0)=0$ and $y(\alpha, \beta, 0)=0.1$. So with respect to these normalizations, in the absence of a predator, the values of $k=0.1,0.4$, and 2.4 represent the prey growing logistically to a carrying capacity of half, double, and twelve times its original value.

\subsection{Driving coexistence from predator extinction}

We first consider $(k, i)$ to be $(0.1,0.1)$. We observe three possible behaviours: predator extinction, seen when both $\epsilon$ and $\eta$ are small, coexistence, and prey extinction which is observed when $\eta$ is approximately greater than or equal to 0.45 .

The case in which the predator becomes extinct is shown in Figure 3. In the absence of the predator, the prey population tends to its carrying capacity $k$, which here is one half of the population's initial size. This was found to be the case for $d=10^{-3}, 10^{-2}, 10^{-1}$, and 1 . For these kinds of equilibrium dynamics, changing the diffusion rate does not appear to drive a qualitative change in the system, although it does have a quantitative effect on the value of this equilibrium.

As can be seen in Figure 4, increasing either $\epsilon$ or $\eta$ can change the nature of the system from predator extinction to coexistence. However, increasing $\eta$ further can cause prey extinction, which is not true for any value of $\epsilon$.

In the case of prey extinction, the predator feeds solely on the subsidy, and in Figure 4 (d), the predator population appears to have a linear dependence on the subsidy conversion rate $\eta$. This can be explained by looking at the governing equations which become simpler when the prey is absent. 


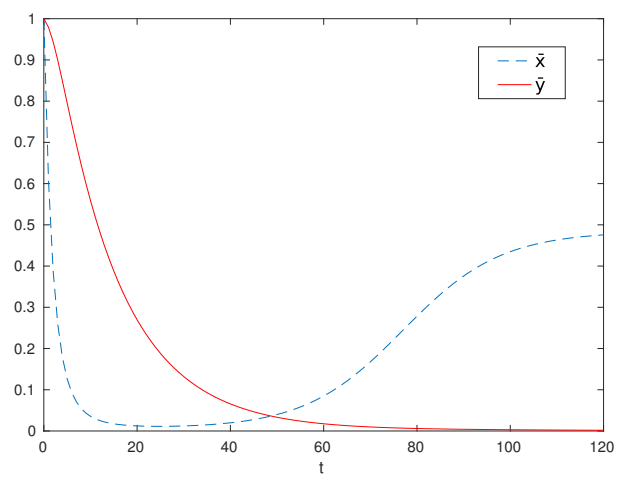

Figure 3: Averaged prey $(\bar{x})$ and predator $(\bar{y})$ populations with $k=0.1, i=0.1, d=0.01, A=0.64, \epsilon=0.1, \eta=0.1$, and on a $100 \times 100$ spatial grid. The predator dies out and the prey population tends to its carrying capacity, which is 0.5 times the prey's initial population.



(a)

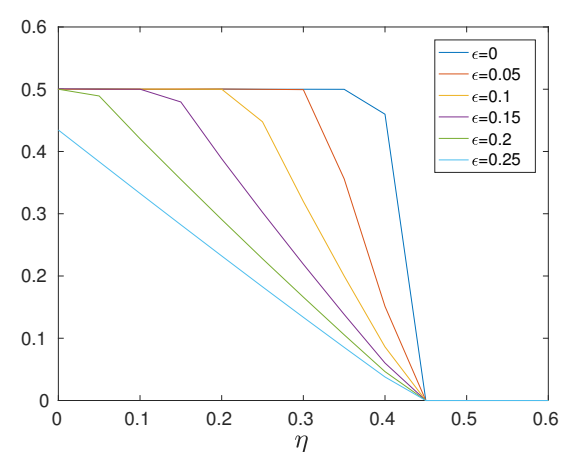

(c)

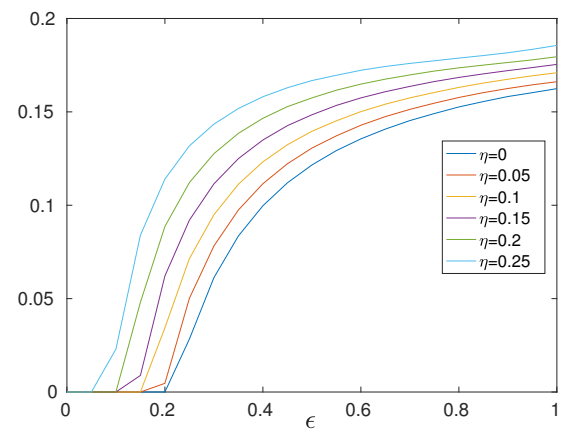

(b)

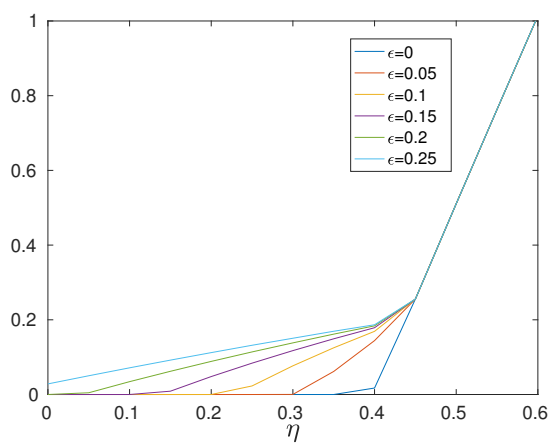

(d)

Figure 4: Averaged equilibrium prey populations in (a) and (c), and predator populations in (b) and (d), plotted against the prey conversion rate $\epsilon$ in (a) and (b) and the subsidy conversion rate $\eta$ in (c) and (d). For all plots, $k=0.1, i=0.1, d=0.01$ and $A=0.5$. The data has been generated using steps in $\epsilon$ and $\eta$ of size 0.05 , and the equilibrium values have been taken after 1000 time steps. 
In the linear section of Figure $4(\mathrm{~d})$, the prey population is zero. So, set $x$ to be zero in Equations (1)-(3). For simplicity, we look for steady spatially uniform states, although we incorporate the effect of $I$ being nonzero only in $\Omega_{0}$ by multiplying the subsidy creation rate $i$ by the relative size of $\Omega_{0}, A$. Then, we have two equations for the subsidy and predator populations.

$$
A i-\gamma s^{*}-\psi\left(\frac{s^{*}}{s^{*}+c}\right) y^{*}=0, \quad \eta \psi\left(\frac{s^{*}}{s^{*}+c}\right) y^{*}-\delta y^{*}=0 .
$$

Solving these for the steady states $s^{*}$ and $y^{*}>0$ gives

$$
s^{*}=\frac{\delta c}{\eta \psi-\delta}, \quad y^{*}=\eta\left(\frac{A i}{\delta}-\frac{c \gamma}{\eta \psi-\delta}\right) .
$$

In Figure $4(\mathrm{~d})$, the linear $A i \eta / \delta$ term dominates in the right side of the graph (i.e. for $\eta>0.45$ ). As we have normalized the prey population by the initial condition $y(\alpha, \beta, 0)=0.1$, the slope of this line is approximately $0.5=A$, which makes sense as $i / \delta=1$. The subsidy equilibrium is consistent with this linear behaviour in $\eta$, decreasing for large values of $\eta$. Hence the linear dependence of the predator populations on the subsidy conversion rate $\eta$ is a consistent prediction of the model in the absence of any prey.

\subsection{Coexistence equilibria}

When $k=0.4$ and $i=0.3$, we see either coexistence between the populations, or prey extinction. For every combination of parameters examined, the populations exhibited some initial transient oscillation, such as in Figure 5. For some values of $\eta$ and $\epsilon$ we were able to extend these damped oscillations for long periods of time, but we were not able to observe limit cycles for any parameter set for which $k=0.4$ and $i=0.3$.

Figure 6 shows the effects of changing the area on which subsidy is introduced into the system, while keeping the total amount of subsidy increase in $\Omega$ constant. Increasing the rate of diffusion of the predator and prey changes the quantitative effects of increasing $A$, but the qualitative effects stay the same. In particular we note that there is a non-monotonic effect of changing $A$ for the case of small diffusion, $d=0.001$. In Figure 7 we show the spatial distributions of the prey, subsidy, and the predator. Because of the small diffusion rate of the prey and the predator, there appears to be a strong separation between these two populations induced by the presence of the subsidy. The prey appear to thrive away from the subsidy, and hence away from the predator population, for the specific choice of parameter values taken. In particular, the parameter values correspond to a case where the subsidy is more desirable than the prey.

\subsection{Limit cycles}

When $k=2.4$ and $i=0.1$ we observe limit cycles. As seen in Figure 8 , increasing $\eta$ can change the behaviour of the system from a limit cycle into an equilibrium. Increasing $\epsilon$ in general increases the amplitude of the limit cycles to a point depending on $\eta$, after which the amplitude shrinks, eventually reaching an equilibrium. In particular, as each amplitude shrinks to zero, we then recover the positive equilibrium for both predator and

\begin{tabular}{c|c|c}
$d$ & Prey equilibrium & Predator equilibrium \\
\hline $10^{-3}$ & 0.2195 & 0.2383 \\
$10^{-2}$ & 0.2669 & 0.1773 \\
$10^{-1}$ & 0.3067 & 0.1751 \\
1 & 0.3108 & 0.1749 \\
10 & 0.3113 & 0.1750
\end{tabular}

Table 1: Equilibrium values of the averaged prey and predator populations with $k=0.4, i=0.3, A=0.64, \epsilon=\eta=0.1$.

prey. From there, one would need to further modify system parameters to see prey extinction. An example of such limit cycles is seen in Figures 9 - 10.

Fixing $A=0.64$ and $\epsilon=\eta=0.1$, there was no noticeable change in the amplitude or period of the limit cycles when varying the diffusion parameter $d=10^{-3}, 10^{-2}, 10^{-1}$, and 1 . However, the spatiotemporal clustering shown for the predator in Figure $10(\mathrm{a}-\mathrm{d})$ is an interesting effect of considering the spatial domain. While the predators predominantly aggregate in the region of high subsidy around the boundary of the region $\Omega$, when the prey population is high they seem to migrate to the center of the domain to take advantage of this, before leaving and clustering again along the boundary. This effect can be tuned based on parameter choices, so some parameters will give a more pronounced effect, while for others the effect may not be noticeable.

\section{Results and Discussion}

Many of the results presented in Section 3 agree with those found in the two-patch model of [16], in which the prey and subsidy are separated, and the predators move between the two. For example, when $k=0.1, i=0.1, \epsilon=0.1$ and $\eta=0.1$, a predator-free equilibrium is predicted. Our results give weight to the accuracy of the two-patch model, as even in the more realistic but more complicated case of continuous movement, similar results were found. Specifically, compare Figures 3 and 5 in [16] with Figure 4.

Increasing the diffusion of the prey and the predators in our model caused no changes in the asymptotic behaviour of the populations for the parameters tested. At pairs of $(k, i)$ closer to a bifurcation point, it may be the case that increasing diffusion does lead to a qualitative change in long term behaviour. Looking at Table 1, we see that in the case of coexistence, an increase in diffusion leads to an increase in prey populations, and a decrease in predator populations. As shown in Figure 7, when diffusion is low, the predator populations are highest at the edges of the square region. As diffusion increases, the predators spread out into the centre of the region, where there is a higher density of prey. The predators can sustain higher populations when feeding on the subsidy, which is only present at the edge of the region. For these parameter choices, the total predator population is lowest when the predators spread out the most, as they can sustain higher numbers when feeding mostly on the subsidy. If the dispersal rate of the predators is increased, then they are forced by overcrowding to go to less nutritionally 




Figure 5: Averaged prey $(\bar{x})$ and predator $(\bar{y})$ populations with $k=0.4, i=0.3, d=0.1, A=0.64, \epsilon=0.1, \eta=0.1$. After some initial oscillation of the populations, they tend to equilibrium values.

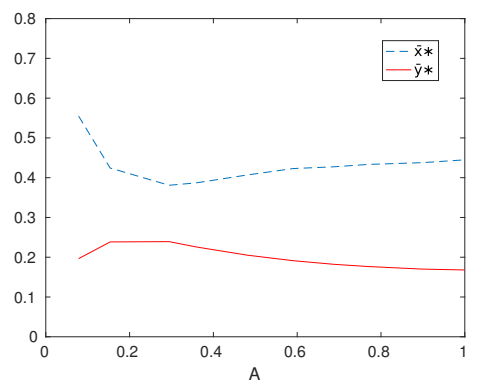

(a)

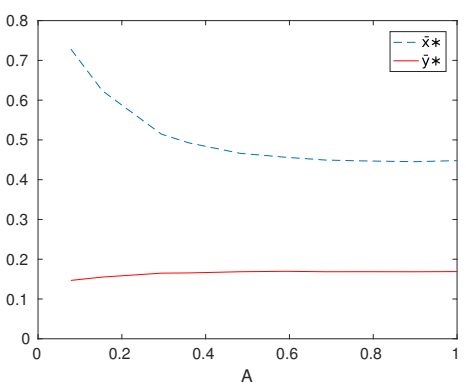

(b)

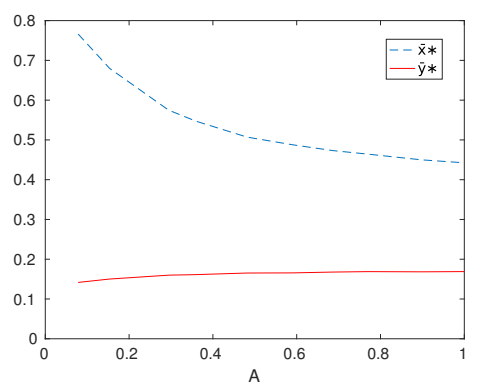

(c)

Figure 6: Averaged equilibrium values of the prey $\left(\bar{x}^{*}\right)$ and predator $\left(\bar{y}^{*}\right)$ populations, plotted against $A$, the ratio of $\left|\Omega_{0}\right|$ to $|\Omega|$. For all plots, $k=0.4, \epsilon=0.1$ and $\eta=0.1$. In order to retain a constant increase in subsidy over the entire region, the rate of subsidy increase $i$ on $\Omega_{0}$ has been scaled such that $i A=0.1445$ for all $A$. (a) $d=0.001$. (b) $d=0.01$. (c) $d=0.1$.

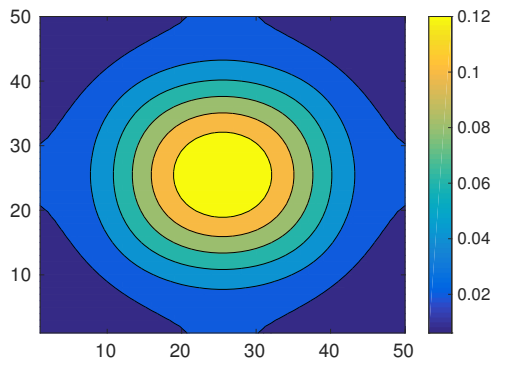

(a)



(b)

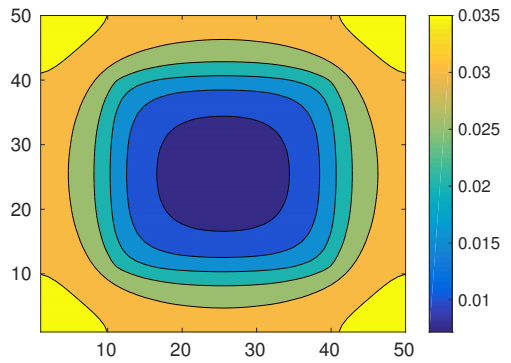

(c)

Figure 7: Spatial plots of equilibrium (a) prey, (b) subsidy and (c) predator populations on a $50 \times 50$ grid, with $k=0.4, i=0.3, A=0.64, \epsilon=0.1, \eta=0.1$ and $d=0.001$. 


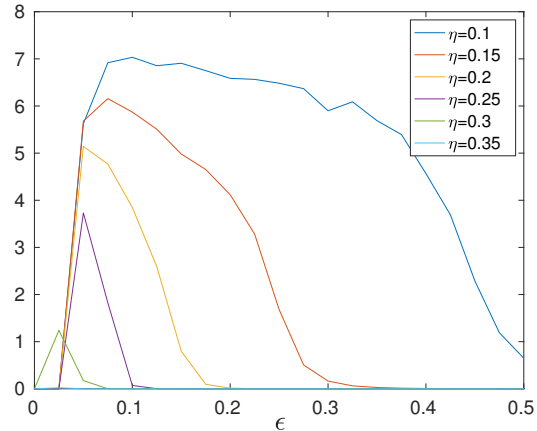

(a)

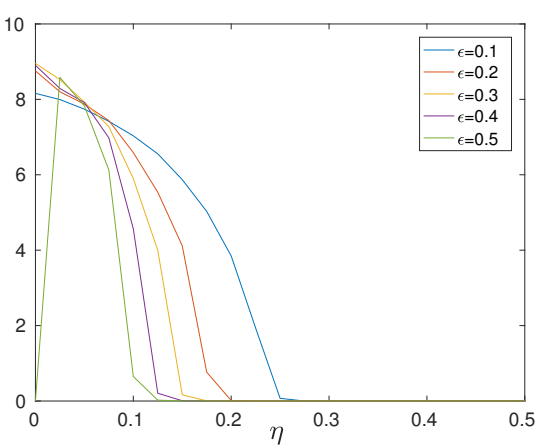

(c)



(b)

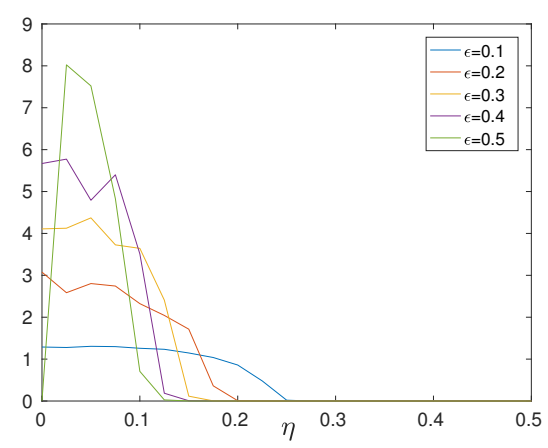

(d)

Figure 8: Limit cycle amplitudes for the averaged prey populations $\bar{x}$ in (a) and (c), and predator populations $\bar{y}$ in (b) and (d), plotted against the prey conversion rate $\epsilon$ in (a) and (b) and the subsidy conversion rate $\eta$ in (c) and (d). For all plots, $k=2.4, i=0.1, d=0.01$ and $A=0.5$. The data has been generated using steps in $\epsilon$ and $\eta$ of size 0.025 , and the amplitudes have been calculated by taking the maximum and minimum populations between times of $t=9000$ and $t=10,000$.

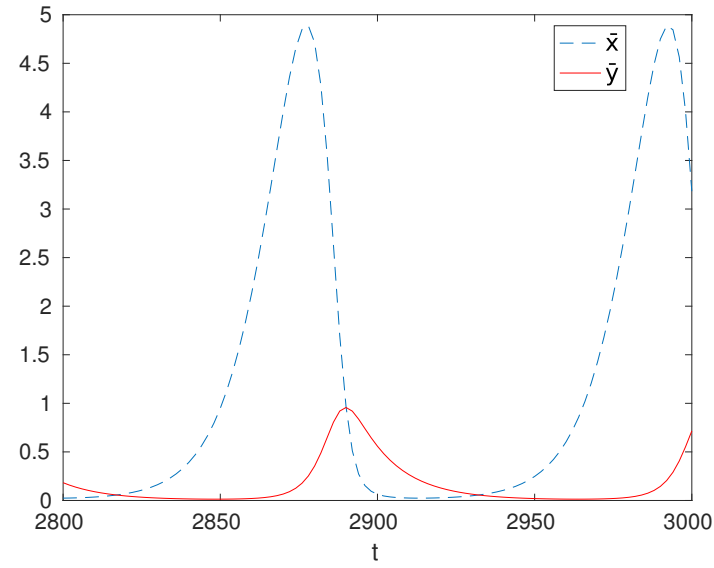

Figure 9: Averaged prey $(\bar{x})$ and predator $(\bar{y})$ populations in a limit cycle. Here, $k=2.4, i=0.1, d=0.001, A=0.64, \epsilon=0.1, \eta=0.1$, and a $100 \times 100$ spatial grid has been used.



(a) $t=2880$



(c) $t=2896$

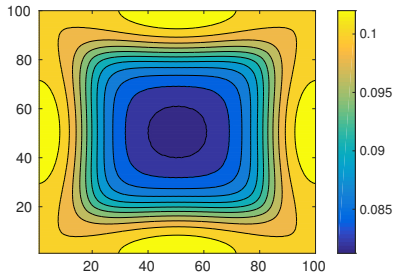

(b) $t=2888$

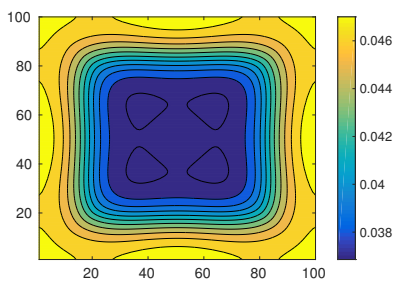

(d) $t=2904$
Figure 10: Spatial plots of the predator population $y$ at various times, corresponding to the limit cycle shown in Figure 9. Here, $k=2.4, i=0.1, d=0.001$, $A=0.64, \epsilon=0.1, \eta=0.1$, and a $100 \times 100$ spatial grid has been used. 
dense locations, and hence are unable to maintain a higher population against their ambient death rate $\delta$.

In all cases, increasing $\eta$ sufficiently had the effect of eradicating the prey, which agrees with results in [6] and [16]. An increase in $\eta$ increases the number of predators, as the nutritional value of their alternative food source is higher. When predator populations become sufficiently large, prey populations die out, and due to the high conversion rate $\eta$, the predators are able to survive without the prey. This is dependent on the rate at which the populations diffuse. If the predators can only move very slowly, then the increased number of predators caused by a highly nutritional subsidy cannot 'spill-over' into the region in which the prey numbers are highest.

In Figure 6, we see that increasing the area on which subsidy numbers increase leads to a decrease in prey numbers and an increase in predator numbers when diffusion is sufficiently high. The quantitative values of these equilibria, and their spatial distributions, are dependent on the geometry under consideration. Circular patterns shown in Figure 7 are likely due to a competition between dispersal forces and the kinetic effects of predators seeking denser nutritional sources, and prey surviving only when they have successfully avoided predation.

In the case of arctic foxes and lemmings, the area on which seal carcasses are deposited could become smaller if the volume of sea ice around coastal arctic regions decreases. This would have a negative impact on fox populations, but may lead to an increase in lemming numbers. However, a decrease in sea ice has been observed to lead to a decrease in seal and polar bear numbers $[3,18]$, so in the case of the arctic, it may be difficult to separate the effects of a decrease in subsidy area and a decrease in subsidy numbers. The model presented here could be used to disentangle these effects by understanding the relative importance in the area the subsidy is present in $A$, and the rate in which the subsidy is deposited $i$.

Three to four year cycles in lemming populations have been observed [22, 10,9], so it is of particular interest to us to note what factors could destroy a limit cycle in the populations. From Figure 8, an increase or decrease in the nutritional value to arctic foxes of lemmings could destabilise the limit cycles. In contrast, an increase in the conversion rate of seal carcasses could result in a collapse of the limit cycles, pushing the system into the positive steady state, but a decrease could not. It was also found that changing the rate of diffusion did not have an effect on the amplitude or period of the limit cycles, which suggests that restricted or enhanced movement of one or both of the predator and prey will not change the gross behaviour of the populations. However, in Figure 10 the spatial distributions of the predator population during these oscillations have an interesting shape that is related to the given geometry of the domain, and of the subsidy. This is a feature of a spatially continuous domain in that we can study nontrivial spatiotemporal oscillations that depend on the geometry of the domain and the relationship it has with the continuous Laplacian representing species dispersal.

Although our model showed qualitatively similar behaviour to that in [16], it demonstrates unique features, such as continuous aggregation, not present in spatially discrete models. In our model, all populations are able to move freely in $\Omega$, whereas in [16], the prey and subsidy are contained in separate spatially homogeneous patches. Although the subsidy numbers can only increase inside $\Omega_{0}$ in our model, which suggests that $\Omega_{0}$ would be a sensible analogy to one of the two patches, the prey are also able to move into this region, and the dispersal is dependent on the local population gradients, rather than the bulk population size in each region. In the two-patch model, the rate of movement is entirely dependent on the difference between the total populations in the two patches, and hence more detailed spatial dispersal is not captured.

\section{Conclusions}

Models in which two species of prey exist in separate patches, with a common predator able to move between the patches, were developed in [6], and used as a way to explain coexistence between two distinct species of prey. The application of a two patch model to systems in which a predator may feed on its preferred prey or on a resource subsidy was considered in [16]. It was predicted that prey extinction was less likely in the two patch model, as higher predator populations caused by plentiful subsidy supply took time to 'spill-over' into the patch containing the prey. In the spatially continuous model, it was found that in certain cases, increasing the diffusion rates of the predator and prey populations led to a higher number of prey. This seems to suggest that if the predator populations spread out across the region too much, the potential benefits of having a plentiful subsidy will not be reaped.

According to our model, a decrease in the size of the region on which the resource subsidy is deposited could have a positive effect on prey populations, provided that the total amount of subsidy deposited per unit time remains fixed. This is an example where the more complicated model defined over a continuous spatial domain can give insights lost by a two patch model (or, general discrete multipatch models), as we can observe the effects of continuously varying this area.

In the case where limit cycles already exist, variation of conversion efficiency parameters can modify or even collapse the limit cycle emergent under the paradox of enrichment. However, we do not observe any cases where there would not typically be limit cycles which were pushed into limit cycles by a variation of conversion efficiency parameters. Therefore, the variation of conversion efficiency apparently can modify the paradox of enrichment for appropriate values of carrying capacity $k$ already giving limit cycles. This is seen in Figure 8, where the amplitude of the limit cycles arising from the paradox of enrichment is modified. For large values of either conversion efficiency parameter, the limit cycle may be suppressed, pushing the system back into a coexistence equilibrium.

In a qualitative sense, many of our results are comparable to those found in previous work, giving support to the strength of ODE models for analysing predator-prey-subsidy systems. The continuous spatial domain model could, in theory, be used to look closely at spatial behaviour and patterning of the populations, and we expect this to play a significant role when the geometry of the regions the populations inhabit are complicated, 
or in the case of a subsidy input rate $I$ with high spatio-temporal variability. Even for our simple square domain with piecewiseconstant subsidy input rate, we observed nontrivial spatial patterning which could lead to more complex migratory and boomand-bust behaviours not readily observed in $n$ patch models.

\section{References}

[1] P. Chesson. Making sense of spatial models in ecology. Modeling spatiotemporal dynamics in ecology, pages 151-166, 1998.

[2] H.N. Comins and M.P. Hassell. Predation in multi-prey communities. Journal of Theoretical Biology, 62:93-114, 1976.

[3] S.H. Ferguson, I. Stirling, and P. McLoughlin. Climate change and ringed seal (Phoca hispida) recruitment in western Hudson Bay. Marine Mammal Science, 21:121-135, 2005.

[4] K. Frafjord and P. Prestrud. Home range and movements of arctic foxes Alopex lagopus in Svalbard. Polar Biology, 12:519-526, 1992.

[5] E. Harvey, I. Gounand, P. Ganesanandamoorthy, and F. Altermatt. Spatially cascading effect of perturbations in experimental meta-ecosystems. Proceedings of the Royal Society of London B: Biological Sciences, 283(1838), 2016.

[6] R.D Holt. Spatial heterogeneity, indirect interactions, and the coexistence of prey species. The American Naturalist, 124:377-406, 1984.

[7] P. Kareiva, A. Mullen, and R. Southwood. Population dynamics in spatially complex environments: Theory and data [and discussion]. Philosophical Transactions: Biological Sciences, 330(1257):175-190, 1990.

[8] A.N. Kolmogorov. Sulla teoria di Volterra della lotta per l'esistenza (on Volterra's theory for the struggle for existence). Giornale dell Istituto Italiano Degli Attuari, 7:74-80, 1936.

[9] C.J. Krebs. Population cycles revisited. Journal of Mammalogy, 77:8-24, 1996

[10] C.J. Krebs and J.H. Myers. Population cycles in small mammals. Advances in Ecological Research, 8:267-399, 1974.

[11] D. Levy, H.A. Harrington, and R.A. Van Gorder. Role of seasonality on predator-prey-subsidy population dynamics. Journal of Theoretical Biology, 396:163-181, 2016.

[12] R.M. May. Limit cycles in predator-prey communities. Science, 177:900902, 1972.

[13] R.M. May. Biological populations with nonoverlapping generations: stable points, stable cycles, and chaos. Science, 186:645-647, 1974.

[14] W.W Murdoch. Switching in general predators: experiments on predator specificity and stability of prey populations. Ecological Monographs, 39:335-354, 1969.

[15] W.W. Murdoch and A. Oaten. Predation and population stability. Advances in Ecological Research, 9:1-131, 1975.

[16] A.L. Nevai and R.A. Van Gorder. Effect of resource subsidies on predatorprey population dynamics: a mathematical model. Journal of Biological Dynamics, 6:891-922, 2012.

[17] M. Predavec and C.J. Krebs. Microhabitat utilisation, home ranges, and movement patterns of the collared lemming (Dicrostonyx groenlandicus) in the central Canadian Arctic. Canadian Journal of Zoology, 78:18851890, 2000.

[18] E.V. Revgehr, N.J. Lunn, S.C. Amstrup, and I. Stirling. Effects of earlier sea ice breakup on survival and population size of polar bears in western Hudson Bay. Journal of Wildlife Management, 71:2673-2683, 2007.

[19] A. D. Rodewald, L. J. Kearns, and D. P. Shustack. Anthropogenic resource subsidies decouple predatorprey relationships. Ecological Applications, 21(3):936-943, 2011.

[20] J.D. Roth. Temporal variability in arctic fox diet as reflected in stablecarbon isotopes; the importance of sea ice. Oecologia, 133:70-77, 2002.

[21] L.F. Shampine and M.W. Reichelt. The MATLAB ODE Suite. SIAM Journal on Scientific Computing, 18:1-22, 1997.

[22] N.C. Stenseth. Population cycles in voles and lemmings: density dependence and phase dependence in a stochastic world. Oikos, 87:427-461, 1999.

[23] D. Tilman and P. M. Kareiva. Spatial ecology: the role of space in population dynamics and interspecific interactions, volume 30. Princeton University Press, 1997.
[24] A.M. Turing. The chemical basis of morphogenesis. Philosophical Transactions of the Royal Society of London. Series B, Biological Sciences, 237:37-72, 1952.

[25] P. Turner and A.D. Taylor. Complex dynamics in ecological time series. Ecology, 73:289-305, 1992.

[26] M. van Baalen, V. Křivan, P.C.J. van Rijn, and M.W. Sabelis. Alternative food, switching predators, and the persistence of predator-prey systems. The American Naturalist, 157:512-514, 2001.

[27] V. Volterra. Variations and fluctuations in the number of individuals in animal species living together. Animal Ecology, 1931. 